\title{
El pulso
}

electromagnético

(PEM): La energía más destructiva

M.Sc. Eduardo Mendieta R.

Profesor de Física y

Teoría Electromagnética

UPS-sede Guayaquil 
Los efectos del pulso electromagnético (PEM) fue experimentado en los años 40 luego de las pruebas de la primera bomba atómica, cuando los equipos de las estaciones de radio y los de tipo eléctrico fueron completamente inutilizados en Hawai ubicado a unos $1200 \mathrm{~km}$ del sitio de pruebas. Pero debido a la falta de investigación sobre el tema, el efecto real del PEM fue entendido recién en 1962 cuando los Estados Unidos realizó una serie de pruebas atmosféricas de gran altitud que trajo como consecuencia el tratado firmado en 1963 con Rusia al cual se lo denominó el tratado de Pruebas Atmosféricas Prohibidas.

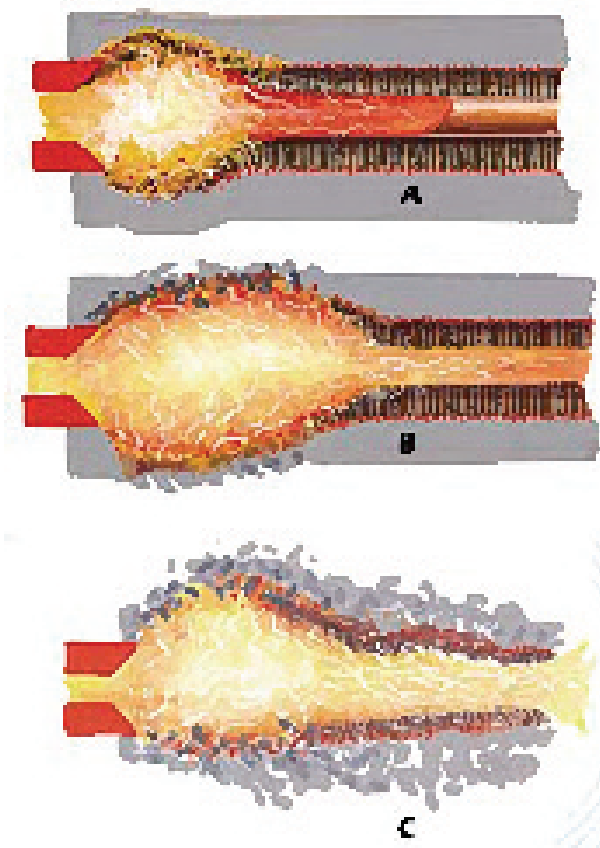

Figura 1.- (A) la explosión expande el tubo, cortocircuitando una bobina y comprimiendo el campo EM hacia fuera (B). El pulso es emitido en (C). Revista Mecánica Popular 2001

Desafortunadamente, el potencial destructivo de un PEM incrementa con el creciente desarrollo tecnológico a medida que la sociedad se vuelve más dependiente de los equipos electrónicos. El PEM no causa daños en la mayoría de las personas pero puede poner en peligro la vida de aquellos que usan marcapasos o algún otro dispositivo electrónico implantado en el cuerpo.
Un PEM es creado cuando un artefacto nuclear es detonado sobre la atmósfera terrestre y actúa más rápido, más breve y más fuerte que el golpe de un rayo, siendo invisible y sin ruido aparente. Sin embargo, este pulso de corta duración crea corrientes de alta intensidad y sobrevoltajes en los equipos electrónicos conectados a una fuente de poder o que utilizan antenas. Esto incluye a los equipos de comunicaciones, computadoras, artefactos electrodomésticos, automóviles modernos, aviones y helicópteros con sistemas electrónicos de ignición y conducción. El daño se produce en los componentes electrónicos basados en materiales semiconductores, tales como memorias, procesadores, integrados, así como en transistores y elementos de alta potencia como Triacs, tiristores, etc. Estos elementos resultan quemados por el paso breve de corriente de gran intensidad y por ende el equipo queda completamente inutilizado, por lo que podría originar incluso muchos accidentes y muertes circunstanciales en el caso de que se produzca un PEM al momento de que un vehículo este siendo utilizado.

Una detonación PEM puede destruir equipamiento electrónico en un radio de acción de aproximadamente 1500 km si se lo efectúa a gran altitud. Lo siniestro del asunto es que una detonación nuclear de gran altitud produce un flujo inmediato de rayos gamma dentro del artefacto. Los fotones liberados producen a su vez una gran energía en altitudes entre los 20 a $40 \mathrm{~km}$. Estos electrones son atrapados en el campo magnético de la Tierra, dando paso a una corriente eléctrica oscilante incremental oscilante.

La corriente eléctrica es asimétrica y produce un campo electromagnético de crecimiento rápido llamado pulso electromagnético el cual debido a la forma como los electrones son atrapados simultáneamente, es un campo electromagnético coherente muy extenso.

La preocupación mundial de que un ataque terrorista pueda involucrar el uso 


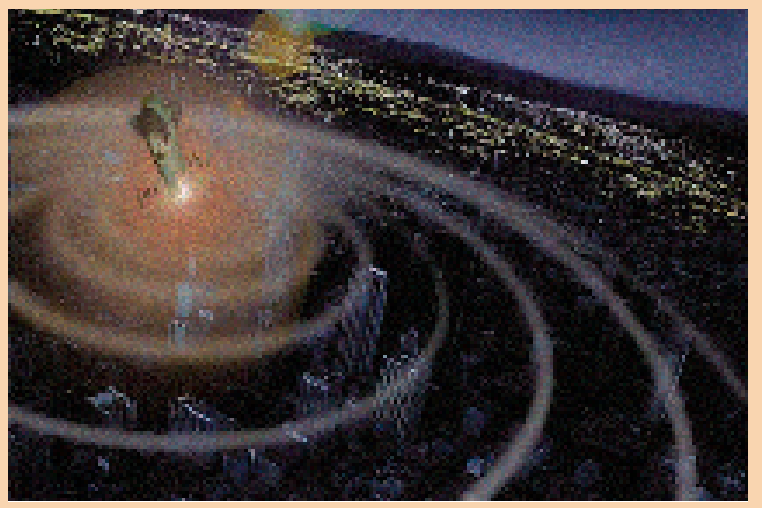

Figura 2.- Propagación de un pulso electromagnético de gran altura. Revista Mecánica Popular 2001

Tabla 1.- Sumario de un PEM

\begin{tabular}{lcc}
\hline Tipo & $\begin{array}{c}\text { Amplitud } \\
\text { Pico }\end{array}$ & $\begin{array}{l}\text { Tiempo } \\
\text { efectivo }\end{array}$ \\
\hline Radiación & $50 \mathrm{KV} / \mathrm{m}$ & $\begin{array}{l}\text { pocos hs } \\
\text { a } 200 \mathrm{hs}\end{array}$
\end{tabular}

Frecuencia $\quad 2$ a $3 \mathrm{~Hz}$

Alcance

15000 km ó más

de bombas-e que generen PEM es tan grande que en los actuales momentos se desarrollan sistemas de aislamiento tipo Jaula de Faraday para protección de equipos electrónicos. Sin embargo, la radiación es tan intensa que logra penetrar en algunos casos el enrejado puesto a tierra.

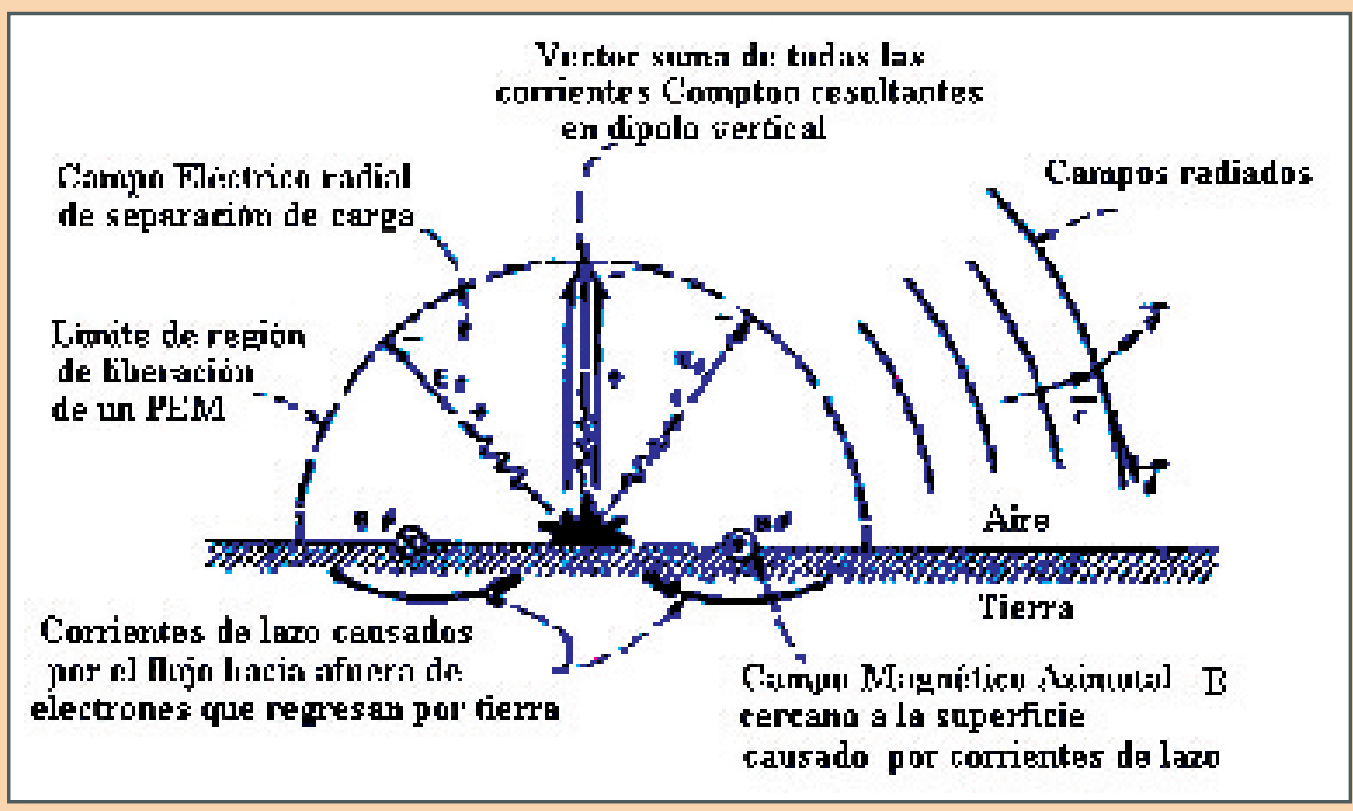

Figura 3 .- Radiación de un pulso electromagnético (PEM) a través de una antena vertical en tierra. 


\section{DAÑO}

(a) Semiconductores. El daño a semiconductores debido al transiente aplicado en un PEM es típicamente alguna forma relacionada de falla termal y por eso está asociada a la energía total instantánea aplicada al dispositivo electrónico. Para elementos discretos (transistores, diodos), el modo de falla predominante aparece localizado en la unión a través de los puntos de soldadura. La soldadura forma trayectos a través de la unión que cortocircuitan o cubren cualquier otra unión en otros elementos del circuito. También se asocia la quemadura de los caminos metálicos de las placas que ocasionan la falla en circuitos integrados. Una exploración aproximada para el análisis de falla es el concepto de rango de falla de poder (Pr). El rango de falla de poder se define como:

$$
P_{r}=A t-b
$$

Donde A es la constante del daño basada en el material del dispositivo y su geometría, y b es la constante de dependencia del tiempo. Estas constantes pueden ser determinadas empíricamente para cada dispositivo de interés mediante datos de pruebas experimentales. Sin embargo, es más conveniente utilizar el modelo de Wunsch, en el que $b=1 / 2$, la ecuación teórica del modelo de Wunsch es:

$$
P_{r}=K^{-1 / 2} \quad k W / c^{2}
$$

Siendo $t$ el ancho del pulso en microsegundos y $\mathrm{K}$ es la constante de daño del modelo de Wunsch en KW - (microsegundos) ? , ya que K representa la potencia necesaria para dañar cierto elemento cuando se aplica un pulso de 1 microsegundo.

(b) Elementos pasivos. Los elementos pasivos más susceptible a ser dañados por una corriente inducida causada por un PEM son aquellos elementos de precisión de baja potencia o voltajes de operación muy pequeños, para los cuales
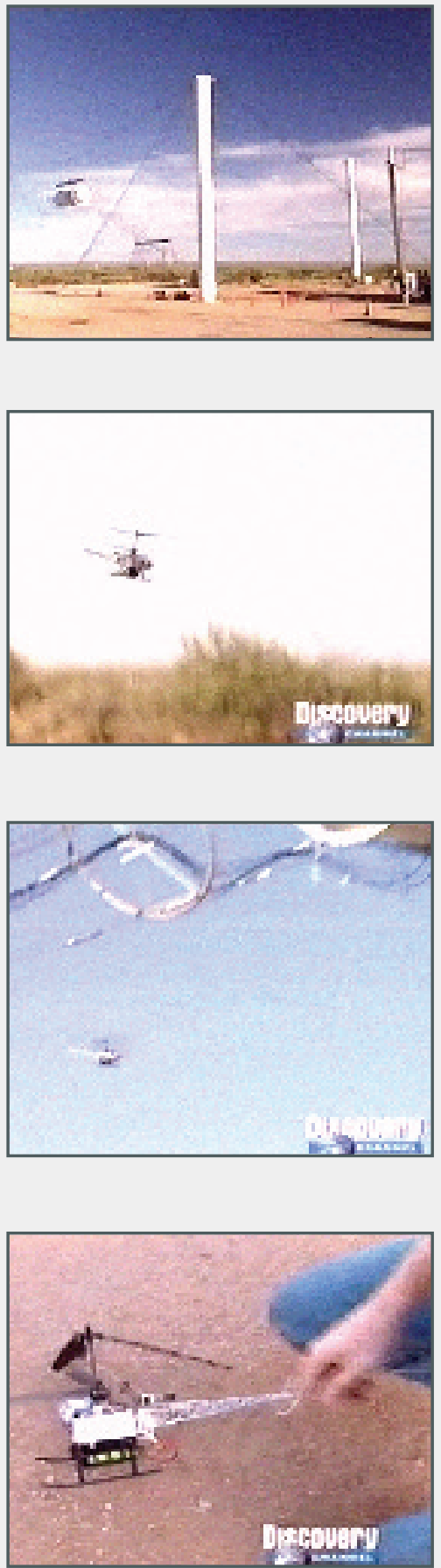

Figura 4.- Prueba secuencial de la efectividad de un PEM. (a) Antena de radiación de pulsos PEM. (b) Helicóptero de pruebas operado control remoto. (c) Helicóptero de pruebas impactado por un pulso PEM radiado por la antena. (d) Helicóptero de pruebas cae inmediatamente al perder su sistema de navegación electrónica. (tomado del Discovery Channel) 


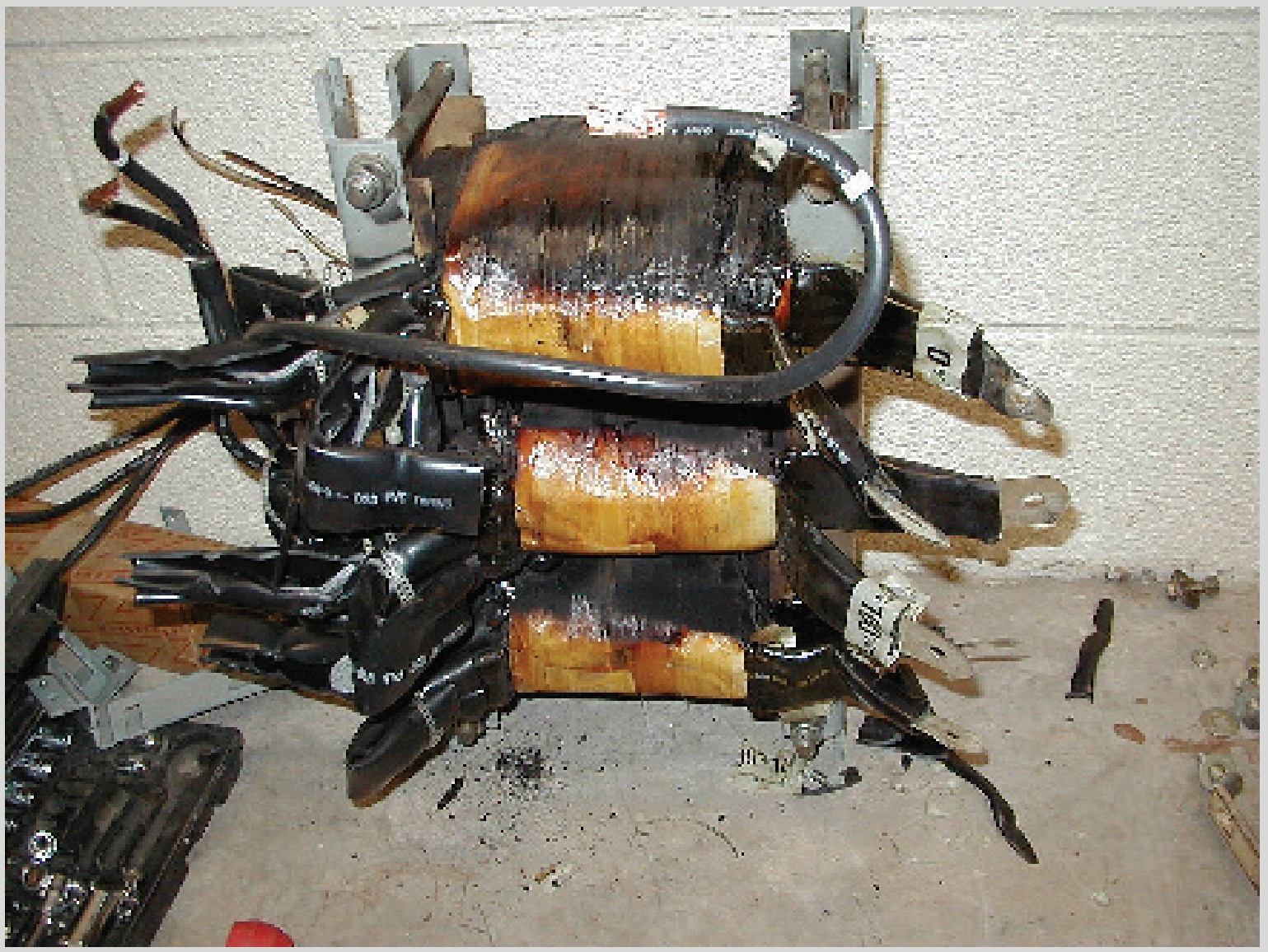

cualquier pequeña variación es significativa. Las resistencias de precisión fallan cuando circulan por ellas corrientes pulsantes de alto nivel que causan un recalentamiento intenso y caída de voltaje. Los capacitores expuestos a corrientes transitorias establecen un voltaje a través del capacitor que incrementa con el tiempo. Para capacitores nanoelectrolíticos el voltaje se mantiene incrementando hasta que se alcanza el nivel de ruptura del dieléctrico. Este nivel de voltaje es aproximadamente 10 veces el nivel normal de operación indicado en las especificaciones del capacitor. Las bobinas y los transformadores pueden dañarse debido a las corrientes inducidas por un PEM que rompen el aislamiento del metal. El punto de rompimiento para el pulso de voltaje es típicamente 5500 voltios para transformadores usados en fuentes de poder y $\mathbf{2 7 5 0}$ voltios para transformadores de pequeña señal.

En conclusión, ante un peligro inminente se debe iniciar de inmediato estu- dios que tiendan a buscar sistemas de aislamientos para los dispositivos electrónicos que representen un peligro de ataque con pulsos electromagnéticos, ya que muchos ataques terroristas están orientados a destruir los sistemas de protección nacional que usan elementos de las Fuerzas Armadas ó en algunos casos centros de cómputos de organismos internacionales. En el caso del terrorismo tecnificado nadie está libre de sufrir un atentado que pudiera incluso poner en riesgo la vida humana.

\section{BIBLIOGRAFÍA}

- http://www.its.bldrdoc.gov/

- http://unitedstatesaction.com/emp_ and_faraday_cages.htm\#other

- http://www.airpower.maxwell.af.mil/ airchronicles/kopp/apjemp.html

- http://www.globalsecurity.org/wmd/ library/report/1988/CM2.htm

- http://www.arrl.org/tis/info/pdf/886 15.pdf

- http://www.discovery.channel.com 